\title{
Nanophotonics
}

SPIEDigitalLibrary.org/jnp

\section{Special Section Guest Editorial: Polaritons}

Ivan A. Shelykh 


\title{
Special Section Guest Editorial: Polaritons
}

\author{
Ivan A. Shelykh ${ }^{\mathrm{a}, \mathrm{b}}$ \\ ${ }^{a}$ University of Iceland, Science Institute, Dunhagi-3, IS-107, Reykjavik, Iceland \\ banyang Technological University, Division of Physics and Applied Physics, \\ 637371, Singapore \\ ishelykh@ntu.edu.sg
}

With the ability to grow multilayered structures down to atomic precision, the age of nanotechnology has begun. In particular, it has now become possible to realize structures with effective confinement of the electromagnetic field interacting efficiently with material excitations, and to achieve a strong coupling regime where the eigenmodes of the system, known as polaritons, have a hybrid half-light, half-matter character.

The apparently simple structure known as a planar semiconductor microcavity evidenced a wealth of different effects. Bosonic statistics have given rise to stimulated scattering, polariton lasing, condensation, and superfluidity. Effective magnetic fields, selection rules, and microscopic interactions describe a rich spin sensitivity and the behavior of spin currents. In threedimensional cavities the quantum nature of the elementary excitations of semiconductor microcavities has yielded quantum squeezing, and the search is on for phenomena such as antibunching and entanglement. Finally, hybrid metal-insulator structures reveal a variety of plasmonic effects which can be used for realization of all-optical integrated circuits.

This special section of the Journal of Nanophotonics comprises a set of selected papers from specialists working in the domain of strong light-matter interactions. The topics covered include nonlinear phenomena in two-dimensional polariton condensates, polariton parametric amplifiers, magnetopolaritons, the Jaynes-Cummings model, intersubband polaritons, and plasmon-polaritons.

0091-3286/2012/\$25.00 @ 2012 SPIE 